\title{
Spatial perception in colliculectomized and normal golden hamsters (Mesocricetus auratus)
}

\author{
JOHN J. KESELICA \\ Albert Einstein College of Medicine, Bronx, New York 10461 \\ and \\ RICHARD R. ROSINSKI \\ University of Pittsburgh, Pittsburgh, Pennsylvania 15260
}

\begin{abstract}
Held and Schneider and others have postulated two, functionally separate, modes of vision-one concerned with localization, the second with identification-and have suggested that the superior colliculus is crucial for the perception of location. Colliculectomized hamsters were tested on two localization tasks. No deficit in depth perception was observed. Perceptual-motor integration necessary to perform a search task was severely disrupted, however. These results suggest that the superior colliculus functions in the control of fine visual-motor coordination.
\end{abstract}

Research by Schneider (1969) has specified anatomical loci for two functional mechanisms of vision in golden hamsters. Colliculectomized subjects in a discrimination task could discriminate simple patterns, but could not orient or locomote directly to the correct pattern. Conversely, animals with ablations of the visual cortex displayed an impaired ability to discriminate patterns, but no loss of ability to orient and localize.

These results, in addition to those of Held (1968), Ingle (1967), and Trevarthen (1968), have led these authors to postulate two different modes of visionthe first concerned with "location" and the second with "identification." The term "location," however, refers to a wide range of behaviors which require the pickup of different types of stimulus information, and which demand varying degrees of visuomotor integrative ability in order to be performed. The question arises that perhaps a lesion in a specific area of the brain could interfere with an animal's performance on one task involving "location" while another behavior that can be categorized under the same heading remains intact. That this may be the case is suggested by previous studies involving ablations of the superior colliculus.

For example, Lashley (1937) demonstrated that rats with lesions in the superior colliculus could negotiate jumps of between 20 and $40 \mathrm{~cm}$ to a platform without any difficulty, and found that the

The authors wish to thank Gerald Schneider for his advice on the surgical procedures used in these experiments, P. A. Cabe for his suggestions concerning the open-field test, and E. J. Gibson for her advice and encouragement. Requests for reprints should be sent to Richard R. Rosinski, Department of Psychology, University of Pittsburgh, Pittsburgh, Pennsylvania 15260. force exerted by the collicular-lesioned animals at the various distances tested was not significantly different from the force used by normal controls tested in the same apparatus. Blake (1959), working with colliculectomized cats on brightness and pattern discrimination tasks, found that her animals displayed normal visual placing responses, and could jump from a tabletop to the floor within a short time after their operations. However, the tectally lesioned animals displayed an inability to visually locate food objects placed before them.

Consequently, in considering an anatomically distinct area as concerned with the perception of layout and location, it is important to determine the role of this area in the pickup of various forms of stimulus information. It is possible that several anatomical areas are concerned with the use of information that specifies location, and that the ablation of one of these areas (e.g., superior colliculus) will affect only certain aspects of localization, leaving others unimpaired. The present experiments evaluate the effect of tectal lesions on the ability to use layout information specifying location and distance of objects relative to the animal. It was hypothesized that the effect of colliculectomy would differ depending on the visual-motor integration necessary for localization.

\section{METHOD}

\section{Subjects}

Fourteen native male golden hamsters (Mesocricetus auratus) were used as subjects. All were between 11 and 14 weeks old at the time of surgery. Six of the hamsters served as controls, and eight as superior collicular lesioned subjects.

\section{Procedure}

Surgery. All lesions were produced according to the procedure 


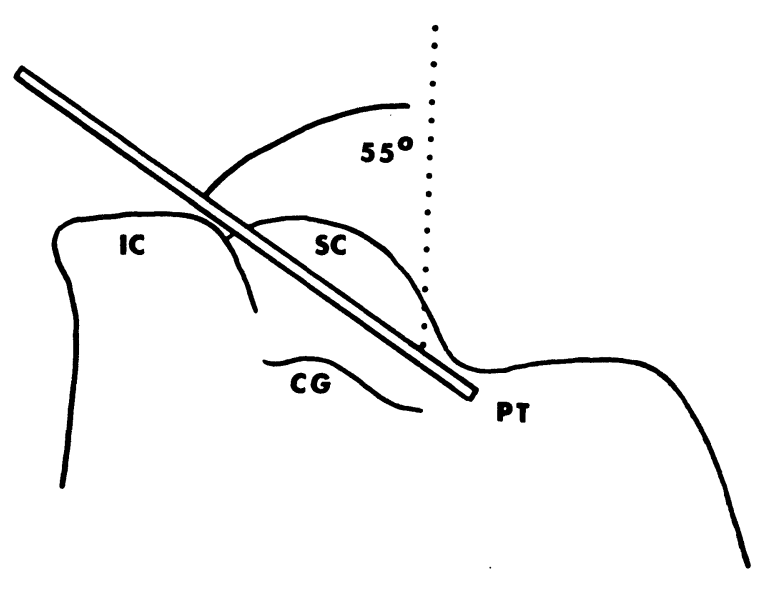

Figure 1. Schematized diagram of surgical incursion. IC, inferior colliculus; SC, superior colliculus; PT, pretectal area; CG, central gray.

described by Schneider (1966). Briefly, the procedures were as follows: (1) Control subjects were given sham operations in which the interparietal bone over both the cerebellus and the inferior colliculus was removed; (2) in experimental subjects, collicular lesions were produced by bilateral undercutting of the colliculus with a specially designed surgical knife (entry at a $55^{\circ}$ angle from the vertical, with the blade passing above the inferior colliculus and periaqueductal gray and into the pretectal area). See Figure 1 (after Schneider, 1969).

Histology. Within a week of the completion of behavioral testing, colliculectomized subjects were perfused through the heart with physiological saline $(0.9 \%)$, followed by $10 \%$ Formalin solution. Frozen frontal sections at $50 \mu$ intervals through the lesioned areas were obtained; every other section was stained with cresyl echt violet solution and examined microscopically to evaluate the extent of the lesion.

Behavioral testing. During the period prior to testing, all subjects were handled and fed sunflower seeds to ensure that each subject would be familiar with the seeds. All behavioral testing was done using a double-blind procedure. A real cliff, a visual cliff, and an open-field test were used. Test order on the real cliff and open-field test was counterbalanced. Testing was done 20-22 days after surgery. Since the possibility existed that some subjects may have used nonvisual information on the real cliff, all subjects were retested on a visual cliff at the end of the experiment.

On the real cliff, the subjects were placed on the centerboard of a real cliff whose near and far sides were covered with a black and white checkerboard pattern at 3-3/8 in. and 25-7/8 in. distance from the centerboard, respectively. Each square of both patterns had the same projective size when viewed from the centerboard (approximately $16^{\circ} 30^{\prime}$ ). Illumination was from below with fluorescent lighting, and was equal $(5 \mathrm{fc})$ on both sides. Each subject was given five trials with an intertrial interval of $15-20 \mathrm{sec}$. Between trials, the centerboard was cleaned with Windex. For each trial, the subjects were placed in different orientations and positions on the centerboard.

Since nonvisual information was potentially available on the real cliff, each subject was given two trials on a visual cliff which was identical to the real cliff with the following exceptions. A sheet of glass was interposed between the subjects and the patterns to eliminate the possibility of olfactory stimulation being used, and the centerboard was raised 2 in. so that the hamster could not obtain tactual information from its vibrissae. The remaining details of the procedure were identical to those used with the real cliff. Between trials, the centerboard and both sides of the glass were cleaned with Windex.

In the open-field test, 16 sunflower seeds were placed $2-2 / 3$ in. apart in a 4 by 4 matrix on a $30 \times 36$ in. vinyl sheet. Each subject was placed 3 in. away from one side of the seed matrix and facing it. The hamsters were run until 15 or more seeds had been gathered or four trials had been run, whichever came first. (A trial was considered terminated when the animal left the vinyl sheet.) Time per trial and number of seeds obtained per trial were recorded. The vinyl sheet was cleaned with Windex between subjects.

\section{RESULTS}

\section{Anatomical}

The sections depicted in Figure 2 are at a level approximately $0.8 \mathrm{~mm}$ anterior to lambda in the frontal plane (Smith \& Bodemer, 1963). The dotted line in each section denotes the lateral and dorsoventral extent of the lesions. As one can see, the lesions are complete in the lateral directions, and remained so until the more rostral portions of the lesions are reached. In two hamsters (10 and 29), the dorsoventral extent of the lesions encroached upon the dorsal portion of the periaqueductal gray; neither of these animals, however, displayed differences in their behavior compared to other colliculectomized hamsters. Figure 3 shows that in all but one subject (16), the lesions did enter the caudal pretectal area to a minor degree. However, while this hamster collected more seeds than did the other experimental animals, its performance was deficient compared to that of the controls.

\section{Behavioral}

The performance of the two groups did not differ in terms of number of descents to the near side of the real and visual cliffs. The percentage of near-side choices on the real cliff was $94 \%$ for the control group and $100 \%$ for the experimental group; for the visual cliff, the corresponding percentages were $92 \%$ and $100 \%$. Colliculectomy does not disrupt localization in terms of the perception of depth at an edge.

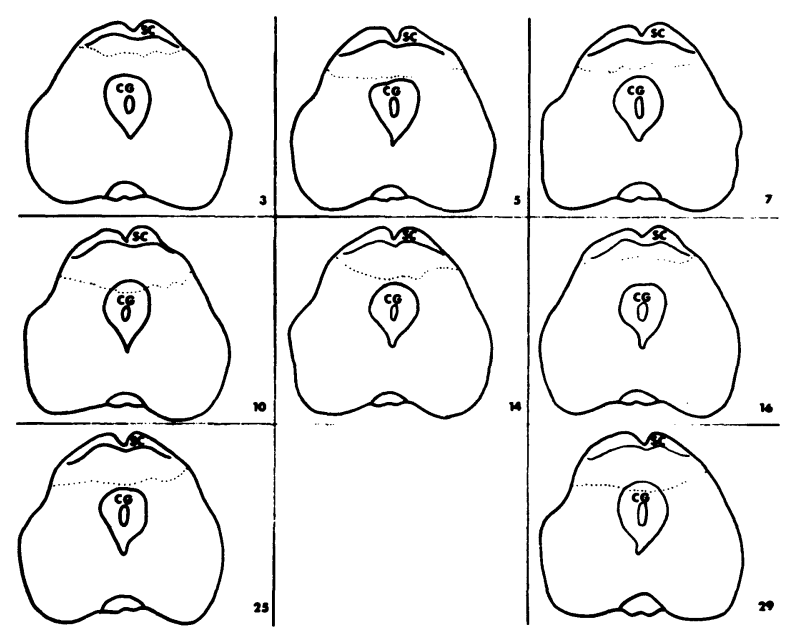

Figure 2. Frontal sections showing extent of collicular lesions. SC, superior colliculus; CG, central gray. 

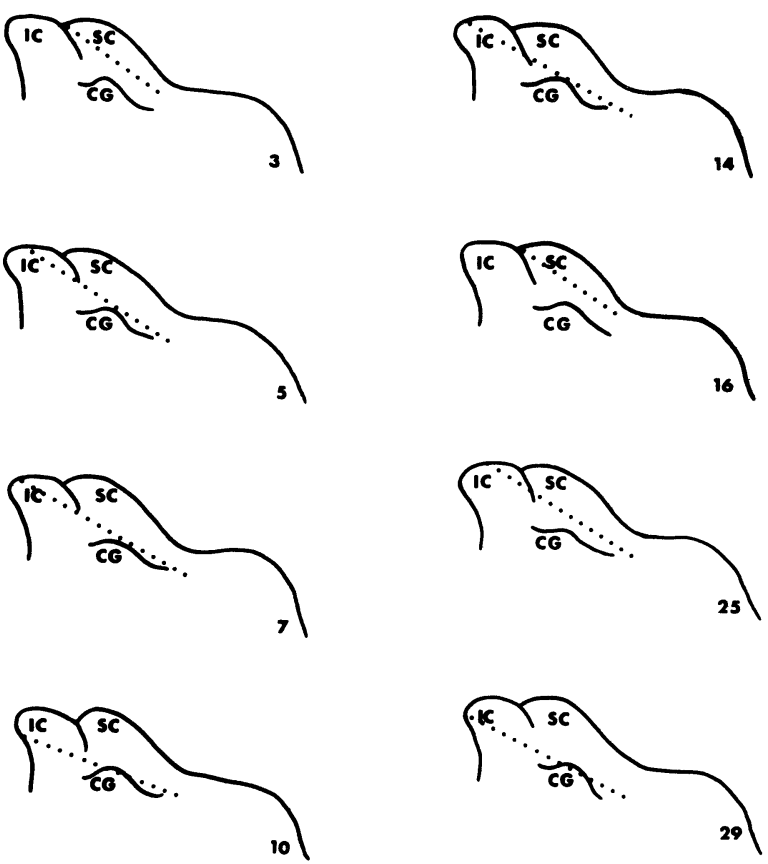

Figure 3. Sagittal reconstruction of collicular lesions. IC, inferior colliculus; SC, superior colliculus; CG, central gray.

Performance of the two groups did differ, however, on the open-field search task. One major difference was that the colliculectomized subjects were totally unsystematic in collecting seeds, whereas normal subjects proceeded quite efficiently. Sample search paths for both groups are shown in Figure 4. The mean number of seeds gathered differed significantly between groups. The experimental group averaged 8.6 seeds, and the control group $15.5(\mathrm{t}=$ $4.25, \mathrm{p}<.01)$. As might be expected from the search paths depicted in Figure 4, the groups differed in search efficiency. Experimental subjects gathered 2.1 seeds per trial and control subjects gathered 11.5 seeds per trial $(t=5.87, p<.01)$.

\section{DISCUSSION}

Ablation of the superior colliculus did not affect perception of depth as measured by the cliff tasks, but it did severely impair visual search. These results demonstrate that the superior colliculus is not a necessary structure for the perception of all aspects of spatial location. Consequently, the dichotomy made by Schneider concerning distinct modes of "identification" and "location" may be an oversimplification. Hein and Held (1967) report other evidence which supports the view that various localization behaviors are dissociable. Restricted rearing of kittens impaired visually guided placing, but not visual-triggered placing. It is clear that at least two (perhaps more) distinct modes of localizing behavior exist.
An analysis of perception of depth in terms of the information available to an active perceptual system reveals a number of possible perceptual modes of "localization." Information for surface distance can be specified in a number of ways. Transformation of the visual information due to head motion parallax can specify depth at an edge.

In a similar fashion, the pattern of motion perspective available to a moving animal specifies distance of a point from the animal. Also, any ability for binocular integration (producing stereopsis) would lead to accurate distance judgments. Finally, an animal must be able to detect a certain amount of nonhomogeneity of surface texture. These types of information appear to be among the major sources used by many animals to determine the location of surfaces in its visual environment. A number of different areas of the brain may be involved in the use of this information specifying surface distance. Removal of only one component of the visual perceptual system would not necessarily result in a deficit on a task like the visual cliff unless all effective depth information is processed in the same region. This seems especially true since the cliff tasks require relatively little fine visual-motor coordination. Once depth has been perceived, the animal need only make a go/no-go discrimination.

In order to perform on the open-field search task, the hamster must not only be capable of perceiving location but maintain the ability to reach an object in an efficient manner. Disruption of either the perception of location or of fine visual-motor coordination would impair search performance. Thus, one could view the hamster's inability to find small objects as revealing a deficiency in the ability to know where an object is; that is the interpretation given by Schneider (1967). On the other hand, one could conclude that the colliculectomized hamster does know where the object is, but is incapable of locomoting directly to it.

The present experimental results suggest that visuomotor deficits may have impaired subjects' search performance. The information specifying distance and position in the cliff tasks (e.g., motion

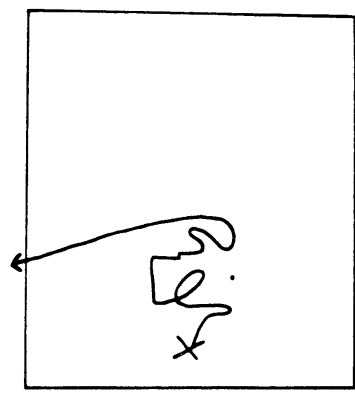

NORMAL

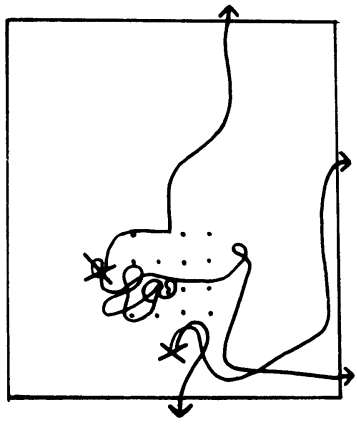

OPERATED
Figure 4. Sample search path of normal and operated animals. 
perspective) also potentially specify distance and position in the search task. Colliculectomized subjects can use this information to guide the relatively gross responses necessary on the cliffs. They cannot do so when the finer responses of search and seed collection are involved. This is further supported by our observations of the hamsters' behavior when seeds were dropped. Although control animals simply picked these seeds up, colliculectomized subjects were unable to find the seed that had just been dropped.

It seems, on the basis of our results, that the superior colliculus of the hamster plays little role in the perception of location, but a large role in the control system underlying fine visual-motor coordination. Attempts to divide vision into distinct, simple modes may be premature at this time. Further analysis of available stimulus information and response control systems will be needed before visualmotor integration can be understood.

\section{REFERENCES}

BLAKE, L. The effect of lesions of the superior colliculus on brightness and pattern discrimination in the cat. Journal of Comparative and Physiological Psychology, 1959, 52, 272-278.
Hein, A., \& Held, R. Dissociation of the visual placing response into elicited and guided components. Science, 1967, 158, 390-392.

HeLd, R. Dissociation of visual functions by deprivation and rearrangement. Psychologische Forshung, 1968, 31, 338-348.

INGLE, D. Two visual mechanisms underlying the behavior of fish. Psychologische Forshung, 1967, 31, 44-51.

LASHLEY, K. S. The mechanism of vision. XIV. Visual perception of distance after injuries to the cerebral cortex, colliculi, or optic thalamus. Journal of Genetic Psychology, 1937, 51, 189-207.

SCHNEIDER, G. E. Superior colliculus and visual cortex: Contrasting behavioral effects of their ablation in the hamster. Unpublished dissertation, M.I.T., 1966.

SCHNEIDER, G. E. Contrasting visuomotor functions of tectum and cortex in the golden hamster. Psychologische Forshung, 1967, $31,52-62$.

Schneider, G. E. Two visual systems. Science, 1969, 163, 895-902.

Smith, O. A., \& Bodemer, C. N. A stereotaxic atlas of the brain of the golden hamster (Mesocricetus auratus). Journal of Comparative Neurology, 1963, 120, 53-64.

Trevarthen, C. B. Two mechanisms of vision in primates. Psychologische Forshung, 1968, 31, 299-337.

(Received for publication July 20, 1976; revision accepted September 3, 1976.) 